\title{
GMR
}

\section{Molecular cloning and evolutionary analysis of captive forest musk deer bitter taste receptor gene $T 2 R 16$}

\author{
G.J. Zhao', N. Wu², D.Y. Li ${ }^{2}$, D.J. Zeng ${ }^{1}$, Q. Chen ${ }^{1}$, L. Lu ${ }^{2}$, X.L. Feng ${ }^{1}$, C.L. Zhang ${ }^{1}$, \\ C.L. Zheng ${ }^{3}$ and H. Jie ${ }^{1}$ \\ ${ }^{1}$ Laboratory of Medicinal Animal, Chongqing Institute of Medicinal Plant Cultivation, \\ Chongqing, China \\ 'Institute of Animal Genetic and Breeding, Sichuan Agricultural University, Ya'an, \\ China \\ ${ }^{3}$ Sichuan Institute of Musk Deer Breeding, Dujiangyan, China \\ Corresponding author: $\mathrm{H}$. Jie \\ E-mail: jiehangisgood@126.com \\ Genet. Mol. Res. 14 (4): 16185-16195 (2015) \\ Received June 18, 2015 \\ Accepted September 20, 2015 \\ Published December 8, 2015 \\ DOI http://dx.doi.org/10.4238/2015.December.8.8
}

\begin{abstract}
Sensing bitter tastes is crucial for most animals because it can prevent them from ingesting harmful food. This process is mainly mediated by the bitter taste receptors (T2R) that are largely expressed in the taste buds. Previous studies have identified some T2R gene repertoires. Marked variation in repertoire size has been noted among species. However, research on T2Rs is still limited and the mechanisms underlying the evolution of vertebrate $T 2 R s$ remain poorly understood. In the present study, we analyzed the structure and features of the protein encoded by the forest musk deer (Moschus berezovskii) T2R16 and submitted the gene sequence to NCBI GenBank. The results showed that the full coding DNA sequence (CDS) of musk deer T2R16 (GenBank accession No. KP677279) was 906 bp, encoding 301 amino acids, which contained ATG start codon and TGA stop codon, with a calculated molecular weight of $35.03 \mathrm{kDa}$ and an isoelectric point of 9.56 . The T2R16 protein receptor
\end{abstract}


had seven conserved transmembrane regions. Hydrophobicity analysis showed that most amino acid residues in T2R16 protein were hydrophobic, and the grand average of hydrophobicity (GRAVY) was 0.657. Phylogenetic analysis based on this gene revealed that forest musk deer had the closest association with sheep (Ovis aries), as compared to cow (Bos taurus), Tursiops truncatus, and other species, whereas it was genetically farthest from humans (Homo sapiens). We hope these results would complement the existing data on T2R16 and encourage further research in this respect.

Key words: Forest musk deer; Bitter taste receptor; T2R16; Structure analysis; Phylogenetic analysis

\section{INTRODUCTION}

Musk deer is a solitary ruminant uniquely distributed in Siberia and Asian countries of Mongolia, China, South Korea, North Korea, India, Burma, Bhutan, and Nepal, especially in the Qinghai-Tibetan Plateau and the Himalayan areas (Groves et al., 1994; Meng et al., 2006; Guha et al., 2007; Sheng and Liu, 2007). China is one of the important range countries that is home to all the recognized species and over $70 \%$ of the world population of musk deer (Yang et al., 2003). Musk deer is $50-60 \mathrm{~cm}$ high at the shoulder, with a brown coat, a pointed face, and large ears. Males have tusk-like upper canines curving down and backwards from the sides of the mouth and a musk gland in the skin of the abdomen. So far, six musk deer species have been reported, namely Siberian musk deer (Moschus moschiferus), forest musk deer (Moschus berezovskii), black musk deer (Moschus fuscus), Alpine musk deer (Moschus chrysogaster), Himalayan musk deer (Moschus leucogaster), and Anhui musk deer (Moschus anhuiensis) (Groves et al., 1994; Li et al., 1999). Among the six species reported in China, forest musk deer has the widest distribution and the largest population (Su et al., 2001; Yang et al., 2003; Sheng and Liu, 2007). Musk deer is famous for musk secreted from the musk glands in males. Musk is major ingredient in many traditional Chinese medicines from several thousand years and has also has been popular essential basis for perfume manufacture (Green, 1986). Data indicates that over $70 \%$ of musk and musk-associated products in the world originate in China (Zou et al., 2005).

Once populated throughout the 13 provinces in China (Groves et al., 1994), musk deer number has declined dramatically in the past decades because of their increased global demand in the medicine and perfume industries. In addition, anthropogenic overexploitation and habitat loss or degradation has caused this species to live on a small number of isolated habitat islands with less space for survival (Yang et al., 2003; Meng et al., 2006; Sheng and Liu, 2007). According to a report released by TRAFFIC, the world's leading wildlife trade monitoring network, and World Wildlife Fund (WWF), musk deer population has reduced sharply from three million in 1950s to no more than a hundred thousand in 1990s. Musk deer are becoming critically endangered with some species difficult to find in the wild. Consequently, all species of musk deer are on the world conservation union IUCN Red List of Threatened Species, as well as in the Appendices of CITES (the Convention on International Trade in Endangered Species of Wild Fauna and Flora). In China, all the musk deer species are now listed as Category I key species under the Wild Animal Protection Law and Chinese legislation of 2002 (Meng et al., 2003).

To save musk deer from extinction and to protect traditional Chinese medicines, the Chinese government has encouraged musk-using enterprises to participate in captive breeding 
program since the early 1950s. In nearly 60 years since the first captive breeding program for forest musk deer was initiated in 1958 many achievements have been made in captive population management, breeding, and prevention of morbidity (Yin and Dai, 1991; Huang et al., 1998; Xu and $\mathrm{Xu}, 2003$ ). Among all species of musk deer, forest musk deer have been the most successful bred and have the largest captive population (Xu and $\mathrm{Xu}, 2003$ ). However, captive population size of forest musk deer is still very small and during the long process of captive breeding, historical records about the genetic backgrounds of many captive populations were either lost or were incomplete. Therefore, understanding the genetic diversity and increasing the size of the captive population are the two most important tasks for breeding managers.

Taste perception refers to sensations triggered by the taste buds on the surface of the tongue, which senses five basic flavors: sweet, salty, sour, bitter, and umami (the taste of monosodium glutamate) (Lindemann, 2001; Yarmolinsky et al., 2009). These gustatory senses are closely related to an animal's diet and play pivotal roles in recognizing conditions of the external world (Glendinning et al., 1999; Chandrashekar et al., 2000). Most taste sensations are triggered via receptor-based sensors expressed in different taste-cell types (Teeter et al., 1992; Nelson et al., 2002; Zhang et al., 2003). Among these basic sensory gustatory modalities, bitter sensitivity has a particularly important role for the survival of animals in the wild. In nature, a variety of toxic substances taste bitter to humans, and virtually all animals show are averse to such tastants (Hilliard et al., 2004; Ueno et al., 2004; Chandrashekar et al., 2006). This suggests that the transduction of bitter taste has potentially evolved as a key defense mechanism against the ingestion of harmful substances. The bitter taste transduction is mainly mediated by bitter taste receptors (T2R) which are encoded by $T 2 R$ genes.

T2Rs are a type of G-protein-coupled receptor (GPCR) distinguished by their seven conserved transmembrane regions (Striem et al., 1989). T2Rs are the largest family of taste receptors, which bind to tastants. Like olfactory and other chemosensory receptor genes, T2R genes also form a multi-gene family and display high sequence similarity (Conte et al., 2002; Shi et al., 2003). Coding regions of T2Rs contain an average of 300 codons, and they are not interrupted by introns, rendering them easy to be detected in whole-genome sequences. Genomic analyses using comparison with reference sequences have revealed the repertoires of $T 2 R$ genes in various vertebrates (Go, 2006; Shi and Zhang, 2006). This family is subject to high rates of genetic variation (Chandrashekar et al., 2006; Bachmanov and Beauchamp, 2007) which is likely to reflect dietary changes during the animal's evolution (Behrens and Meyerhof, 2009). In previous studies, T2R gene repertoires have been uncovered in some mammals, chickens, frogs, and some teleost fishes (Matsunami et al., 2000; Conte et al., 2002, 2003; Parry et al., 2004; Wang et al., 2004; Go et al., 2005; Shi et al., 2003; Shi and Zhang, 2006). The nearly complete human and mouse T2R gene repertoires have been elucidated (Conte et al., 2002, 2003; Shi et al., 2003). Current genome annotation records suggest that there are more than 30 putative functional T2Rs in the mouse genome, while they are fewer in humans, and most have close homologs in mouse (Matsunami et al., 2000; Wu et al., 2005).

Due to the significant role of $T 2 R$ genes in helping animals avoid generally bitter, toxic, and harmful substances, changes in the $T 2 R$ gene repertoire could in turn affect the behavior, especially, the feeding of animals. In the present study aimed to gain insights into evolutionary changes in the $T 2 R$ gene repertoire, we collected a forest musk deer sample and used the molecular biology methods to clone and sequence the T2R16 gene. To generate useful information, we analyzed the structure and characteristics of the T2R16 nucleotide sequence and the protein sequence encoded by the cDNA and conducted a phylogenetic analysis using sequences from other vertebrate 
species. We hope our results would further supplement the molecular and evolutionary research on musk deer taste receptors and provide a scientific basis for musk deer protection and utilization as a genetic resource.

\section{MATERIAL AND METHODS}

\section{Sampling}

Forest musk deer from Chongqing, China was used to obtain the blood sample. Two milliliters blood was collected with a disposable syringe in a tube containing an equal volume of EDTA. It was immediately mixed with the anticoagulant by inverting the collection tube and stored in a refrigerator. The sample was stored at $-70^{\circ} \mathrm{C}$ until it was thawed for analysis. Total DNA in the sample was phenolextracted from serum following standard procedures (Pietro et al., 2011). The animal was treated carefully under as per the ethical guidelines. The protocol was approved by the Committee on the Care and Use of Laboratory Animals of the State-level Animal Experimental Teaching Demonstration Center of Sichuan Agricultural University (Approval ID: Decree No. 10 [2013]).

\section{DNA extraction and clone sequencing}

Total genomic DNA was extracted following the method of Zou et al. (2005). A 586-bp sequence of the mtDNA control region (CR) from the $5^{\prime}$ end was specifically amplified using the primer pair F: 5'-CAAAGCCTAAGCAAATCCTG-3' and R: 5'-GGGCAAACAGA ATGAGGCTT-3'. The primers were designed according to the Sus scrofa (GenBank accession No. XM_003134749.1) and Bos taurus (GenBank accession No. NM_001046628.1) T2R16 sequences. Amplification was performed in a $25-\mu \mathrm{L}$ reaction mixture containing $120 \mathrm{ng}$ template DNA, 1X PCR buffer (Takara, Dalian, China), $0.5 \mathrm{mM}$ dNTPs, $1.5 \mathrm{mM} \mathrm{MgCl}_{2}, 1 \mu \mathrm{M}$ each primer, and $1.5 \mathrm{U}$ Taq polymerase. The reaction was performed in a PTC-100 thermal cycler (MJ Research, St. Bruno, Canada) and the conditions were as follows: $94^{\circ} \mathrm{C}$ for $4 \mathrm{~min}$, followed by 35 cycles of $94^{\circ} \mathrm{C}$ for $30 \mathrm{~s}, 55-59^{\circ} \mathrm{C}$ for 45 $\mathrm{s}$ and $1 \mathrm{~min}$ at $68^{\circ} \mathrm{C}$ for $1 \mathrm{~min}$ with a final extension at $68^{\circ} \mathrm{C}$ for $10 \mathrm{~min}$. The PCR products were electrophoresed on a $1.0 \%$ agarose gel and purified with the DNA Agarose Gel Extraction Kit (Qiagen, Hilden, the Netherlands). Sequencing of purified fragments and the fragments cloned in a $\mathrm{T}$ vector was performed on an ABI 3730xl automatic sequencer (Applied Biosystems, Illumina, USA), and the results obtained from both were compared.

\section{Data analyses}

The obtained sequence was submitted to NCBI Genbank (Accession No. KP677279). Raw sequences were edited and aligned by the program Seqman 5.01 of DNAstar software (DNAstar Inc. Madison, WI, USA). The complete Bos taurus T2R16 sequence was used as the reference sequence for comparing the other T2R16 sequences. Amino acid identity and composition was determined by MEGA 6.0 (Tamura et al., 2007).

Physical and chemical properties of the putative protein were analyzed by Compute pl/Mw tool of ExPASy Proteomics Server; conserved domains of the protein were analyzed by NCBI CDSearch tool; domain and family region was predicted by ScanProsite tool of ExPASy Proteomics Server; transmembrane helices in the protein were predicted by TMHMM Server v. 2.0; hydrophobic property was predicted by protscale of ExPASy Proteomics Server; secondary structure was 
predicted by HNN HNN secondary structure prediction method (https://npsa-prabi.ibcp.fr/cgi-bin/ npsa_automat.pl?page=/NPSA/npsa_hnn.html); phosphorylation loci were predicted by NetPhos 2.0 Server and NetPhosK 1.0 Server; glycation of $\varepsilon$ amino groups of lysine loci were predicted by NetGlycate 1.0 server; predictions of protein function were displayed by the ProtFun 2.2 server.

To ensure that the DNA sequence of the amplified fragment was our target sequence, we carried out a BLAST similarity search in NCBI prior to the sequence analysis. T2R16 sequences of the forest musk deer and other species were edited and aligned using the clustal $x 2$ and DNASTAR package (http://www.dnastar.com/t-dnastar-lasergene.aspx). Kimura 2-parameter genetic distances between forest musk deer and other species were used to construct a Neighbor-Joining (N-J) phylogenetic tree from 1,000 bootstrap replicates with the MEGA6.0 program (Tamura et al., 2007).

\section{RESULTS AND DISCUSSION}

\section{Structure and characteristics of forest musk deer T2R16 protein}

Results showed that the full coding DNA sequence (CDS) of musk deer T2R16 was 906 bp, encoding 301 amino acids, and contained ATG start codon and TGA stop codon. The putative protein had a calculated molecular weight of $35.03 \mathrm{kDa}$ and an isoelectric point of 9.56. We calculated the overall amino acid compositions of the T2R16 protein using MEGA 6.0. The amino acid sequence of the T2R16 protein is presented in Table 1. Total atoms in the T2R16 protein were 5009, including $1659 \mathrm{C}$ (Carbon), $2531 \mathrm{H}$ (Hydrogen), $399 \mathrm{~N}$ (Nitrogen), $405 \mathrm{O}$ (Oxygen), and $15 \mathrm{~S}$ (Sulfur). Formula of T2R16 protein could be written as $\mathrm{C}_{1659} \mathrm{H}_{2531} \mathrm{~N}_{399} \mathrm{O}_{405} \mathrm{~S}_{15}$. Total number of negatively charged residues (Asp $+\mathrm{Glu}$ ) was 13 and the total number of positively charged residues (Arg + Lys) was 23; the positively charged residues were higher than the negatively charged residues. The instability index (II) was computed to be 35.99 denoting that the protein was stable. The estimated half-life of protein was 30 hours in mammalian reticulocytes. The secondary structure of the protein was predominantly 208 a-helices (69.10\%), 30 extended strands (9.97\%), and 63 random coils $(20.93 \%)$. T2R 16 protein was estimated to have one typical conserved domain between amino acids 4 and 299 (E value: 1.11e-71).

Table 1. Amino acid composition of musk deer T2R16 protein.

\begin{tabular}{lcccccccccccccccccccc}
\hline Amino acids & Ala & Arg & Asn & Asp & Cys & Gln & Glu & Gly & His & Ile & Leu & Lys & Met & Phe & Pro & Ser & Thr & Trp & Tyr & Val \\
\hline Number & 18 & 12 & 9 & 4 & 5 & 10 & 9 & 7 & 10 & 21 & 50 & 11 & 10 & 27 & 7 & 32 & 18 & 12 & 8 & 21 \\
Percentage & 6.00 & 4.00 & 3.00 & 1.30 & 1.70 & 3.30 & 3.00 & 2.30 & 3.30 & 7.00 & 16.60 & 3.70 & 3.30 & 9.00 & 2.30 & 10.60 & 6.00 & 4.00 & 2.70 & 7.00
\end{tabular}

The figures represent the total number and percentage of different amino acid residues present in the T2R16 protein.

The results also demonstrated that this receptor had seven conserved transmembrane regions and was a member of G-protein coupled receptor family. Table 2 and Figure 1 show the distribution of amino acid sites on the transmembrane helices of T2R16 protein.

Hydrophobicity analysis showed that most amino acid residues of T2R16 protein were hydrophobic and the grand average of hydrophobicity (GRAVY) was 0.657 (Figure 2).

Five Ser phosphorylation sites were present at positions 7, 221, 222, 274, and 297. One Thr phosphorylation site at position 141 and two Tyr phosphorylation sites at positions 149 and 270 were observed (Figure 3). Meanwhile, we found four Ser PKC phosphorylation sites at 108 (score: 0.68), 140 (score: 0.73), 156 (score: 0.77), and 205 (score: 0.85) positions, respectively, 
and two Thr PKC phosphorylation sites at 141 (score: 0.59) and 216 (score: 0.91) positions. By using NetGlycate 1.0 server, we identified 5 sites for glycation of $\varepsilon$ amino groups of lysine at 106 (score: 0.655), 158 (score: 0.873), 209 (score: 0.842), 288 (score: 0.763), and 292 (score: 0.906) positions, respectively. The predicted results are presented in Figure 4.

Table 2. Prediction of the amino acid distribution on the transmembrane helices.

\begin{tabular}{ll}
\hline Position on the membrane & Distribution of amino acids \\
\hline Outside & $1-9,64-82,152-182,257-260$ \\
TM helix & $10-32,45-63,83-105,129-151,183-205,234-256,261-283$ \\
Inside & $33-44,106-128,206-233,284-301$ \\
\hline
\end{tabular}

TM helix, transmembrane helix.

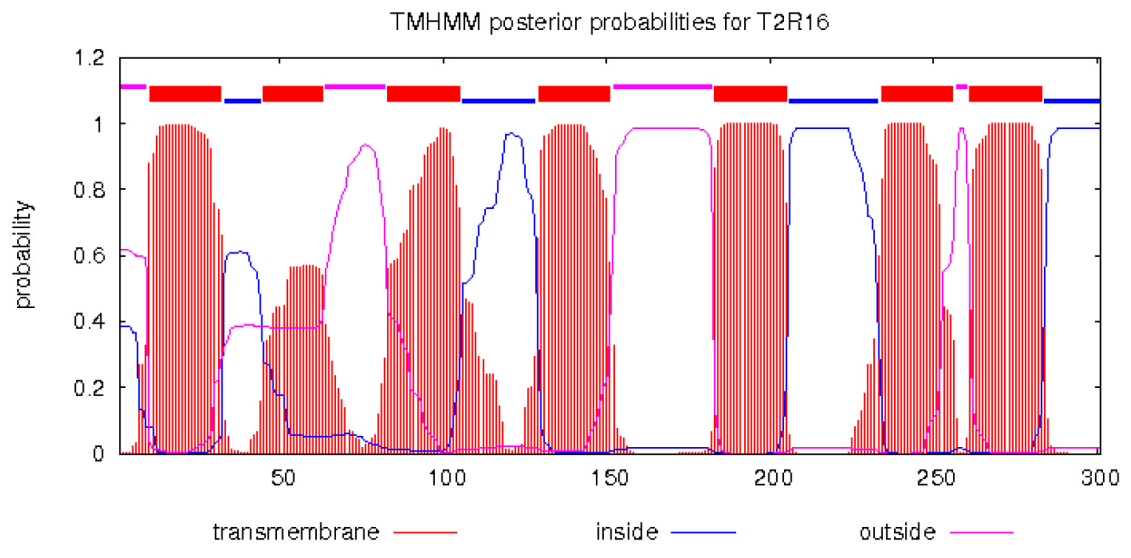

Figure 1. Prediction of transmembrane helices in forest musk deer T2R16 protein by TMHMM Server v. 2.0.

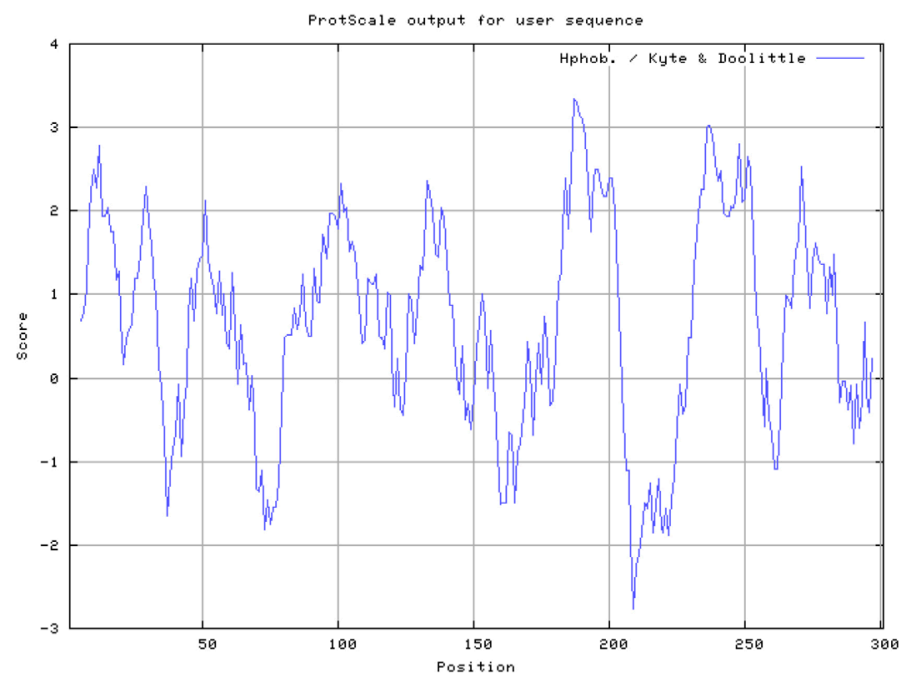

Figure 2. Putative hydrophobicity of forest musk deer T2R16 protein. 
Because studies on the $T 2 R$ genes are scarce and the protein functions and classification are also not well understood, we predicted the T2R16 protein function by using the ProtFun 2.2 server. The prediction results demonstrated that the main function of T2R16 protein was transport and binding (with the highest Odds: 1.990 and the threshold: 0.816) and the gene ontology category was a transporter. These prediction results conform to the characteristics of T2R16 protein with the predicted presence of transmembrane helices in its structure.

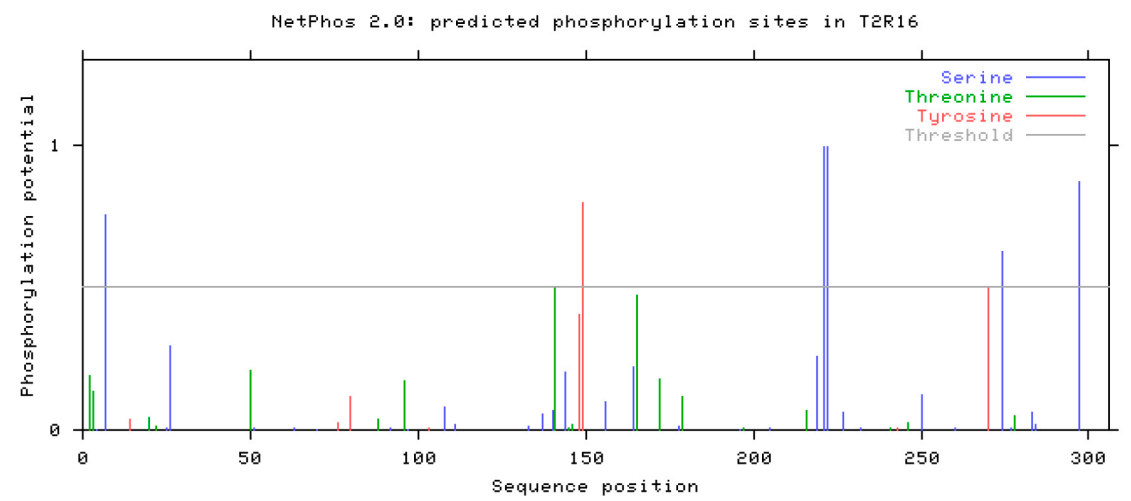

Figure 3. Prediction of musk deer T2R16 protein phosphorylation by NetPhos 2.0.

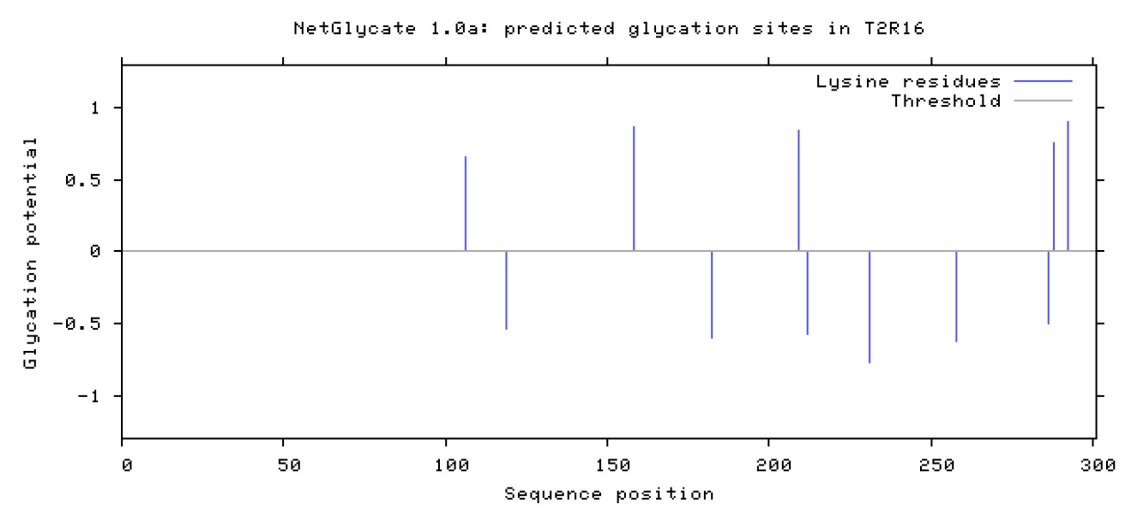

Figure 4. Prediction of glycation of $\varepsilon$ amino groups of lysines in musk deer T2R16 protein by NetGlycate 1.0.

\section{Phylogenetic analysis of T2R16 amino acid sequences of forest musk deer and some other species}

Comparison of the sequences using the NCBI BLAST tool revealed the identity of T2R16 amino acid sequences of forest musk deer and other species (Table 3). The analyses of additional species would be helpful to describe the dynamic evolution of T2R16 gene. A phylogenetic tree was constructed based on the T2R16 sequences between forest musk deer and other animals using the software MEGA 6.0 Neighbor Joining (NJ) method (Figure 5). 
Table 3. Identity of T2R16 amino acid sequences between forest musk deer and other species.

\begin{tabular}{llll}
\hline Species & GenBank ID & Definition & Identity (\%) \\
\hline Bos taurus & NM_001046628.1 & taste receptor, type 2, member 16 (TAS2R16) & 94.48 \\
Ovis aries & XM_004008021.1 & Predicted: taste receptor type 2 member 16-like (LOC101105240) & 94.37 \\
Tursiops truncatus & KJ524798.1 & taste receptor type 2 member 16 (Tas2r16) pseudogene, complete sequence & 85.6 \\
Sus scrofa & XM_003134749.1 & Predicted: taste receptor type 2 member 16-like (LOC100513769) & 81.95 \\
Camelus bactrianus & XM_010947595.1 & Predicted: taste receptor type 2 member 16 (LOC105063129) & 79.58 \\
Equus przewalskii & XM_008514411.1 & Predicted: taste receptor type 2 member 16 (LOC103547299) & 79.58 \\
Camelus dromedarius & XM_010975603.1 & Predicted: taste receptor type 2 member 16 (LOC105085339) & 79.47 \\
Pteropus vampyrus & XM_011357170.1 & Predicted: taste receptor type 2 member 16 (LOC105290162) & 79.14 \\
Myotis brandtii & XM_005878870.1 & Predicted: taste receptor type 2 member 16-like (LOC102256244) & 77.15 \\
Pan troglodytes & NM_001009160.1 & taste receptor, type 2, member 16 (TAS2R16) & 76.03 \\
Homo sapiens & NM_016945.2 & taste receptor, type 2, member 16 (TAS2R16) & 75.28 \\
Macaca mulatta & NM_001080759.1 & taste receptor, type 2, member 16 (TAS2R16) & 75.23 \\
Callithrix jacchus & XM_002752064.2 & Predicted: taste receptor, type 2, member 16 (TAS2R16) & 72.96 \\
\hline
\end{tabular}

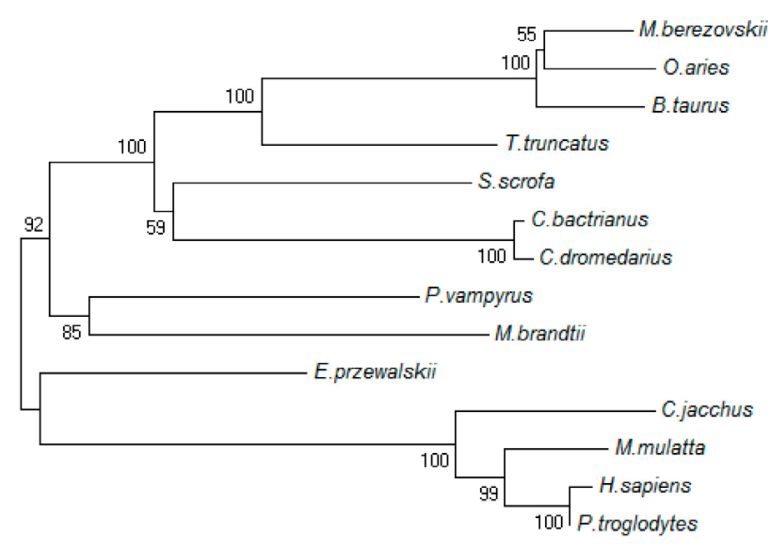

$\longmapsto_{0.02}$

Figure 5. Phylogenetictree generated on the basis of T2R16 amino acid sequences from forest musk deer and other vertebrate species.

The identity among different T2R16 sequences is given in Table 3. As shown in Figure 5, the phylogenetic tree divided these selected vertebrates into two main branches. Homo sapiens were grouped with Pan troglodytes, Macaca mulatta, and Callithrix jacchus with a bootstrap value of $100 \%$; it then formed a clade with Equus przewalskii. The other big clade was formed with forest musk deer and other species, including cow, sheep, pig, and others. Phylogenetic tree can appropriately simulate the evolution process for the considered species. It indicated the feasibility to estimate the evolutionary order according to some gene sequences with important functions, although we cannot determine the evolutionary relationship only according to the sequence of one gene. As is evident from this evolutionary tree, forest musk deer had the closest affiliation with sheep compared to cow, Tursiops truncatus and other species in the big clade and it had the farthest genetic relationship with humans in the other small clade.

Previously, there have been a few reports about $T 2 R$ gene repertoires, and marked variation in repertoire size has been noted among species. The nearly complete human and mouse $T 2 R$ gene repertoires have been elucidated (Conte et al., 2002, 2003; Shi et al., 2003). Some valuable studies of Hu and Shi (2013) and Dong et al. (2009) demonstrated the smallest bitter taste 
receptor (T2Rs) gene repertoire in carnivores and the dynamic evolution of bitter taste receptor genes in vertebrates, respectively. However, few studies on the T2Rs have been undertaken, and the mechanisms underlying the evolution of vertebrate $T 2 R$ s remain poorly understood. In the present study, we attempted some basic analysis of $T 2 R 16$ to provide more theoretical data for research and protection of musk deer. In future, we propose to undertake SNP studies of this gene to pinpoint the relationship between SNPs and evolution in forest musk deer.

\section{CONCLUSIONS}

As an endangered animal group in China, musk deer have greatly attracted the attention of deer-biologists and wildlife conservationists. Clarifying the taxonomic status and distribution of musk deer species is important to determine the conservation status for each species and to establish appropriate conservation strategies. There remains some uncertainty about the species determination of the musk deer in the world. It has long been assumed that bitter taste evolved as a defense mechanism to detect potentially harmful toxins in food. Functional studies have revealed that $T 2 R$ proteins respond to bitter tastants and bitter taste reception is likely to associate with dietary selection (Chandrashekar et al., 2000; Bufe et al., 2002). Thus, species specific T2Rs might be required for animals to detect distinct bitter substances.

In the present study, we analyzed the structure and features of the protein encoded by forest musk deer T2R16 and submitted the gene sequence to the NCBI Genbank. To further explain the genetic basis for this gene, we constructed a phylogenetic tree, which showed the genetic relationship of forest musk deer and some other species based on T2R16, suggesting that forest musk deer had the closest affiliation with sheep compared to cow, Tursiops truncatus, and other species and it had the farthest genetic relationship with humans. Similarly, it suggested $T 2 R 16$ was conserved among vertebrates, and that we can estimate the evolution order based on the phylogenetic tree constructed using sequences of proteins with important functions. Collectively, these results complement the data on $T 2 R 16$, and we hope it will encourage further research on this aspect.

\section{Conflicts of interest}

The authors declare no conflict of interest.

\section{ACKNOWLEDGMENTS}

Research supported by Fundamental Research Funds for the Chongqing Science \&Technology Commission, China (\#2014cstc-jbky-01303, \#2014 cstc-jbky-01302), the National Natural Science Foundation of China (\#81503188), and the Chongqing Research Program of Basic Research and Frontier Technology (\#cstc2015jcyj10116).

\section{REFERENCES}

Bachmanov AA and Beauchamp GK (2007). Taste receptor genes. Annu. Rev. Nutr. 27: 389-414.

Behrens M and Meyerhof W (2009). Mammalian bitter taste perception. Results Probl. Cell Differ. 47: 203-220.

Bufe B, Hofmann T, Krautwurst D, Raguse JD, et al. (2002). The human TAS2R16 receptor mediates bitter taste in response to beta-glucopyranosides. Nat. Genet. 32: 397-401.

Chandrashekar J, Mueller KL, Hoon MA, Adler E, et al. (2000). T2Rs function as bitter taste receptors. Cell 100: 703-711.

Chandrashekar J, Hoon MA, Ryba NJ and Zuker CS (2006). The receptors and cells for mammalian taste. Nature 444: $288-294$. 
Conte C, Ebeling M, Marcuz A, Nef P, et al. (2002). Identification and characterization of human taste receptor genes belonging to the TAS2R family. Cytogenet. Genome Res. 98: 45-53.

Conte C, Ebeling M, Marcuz A, Nef P, et al. (2003). Evolutionary relationships of the Tas2r receptor gene families in mouse and human. Physiol. Genomics 14: 73-82.

Dong D, Jones G and Zhang S (2009). Dynamic evolution of bitter taste receptor genes in vertebrates. BMC Evol. Biol. 9: 12.

Glendinning JI, Tarre M and Asaoka K (1999). Contribution of different bitter-sensitive taste cells to feeding inhibition in a caterpillar (Manduca sexta). Behav. Neurosci. 113: 840-854.

Go Y, Satta Y, Takenaka O and Takahata N (2005). Lineage-specific loss of function of bitter taste receptor genes in humans and nonhuman primates. Genetics 170: 313-326.

Go Y (2006). Proceedings of the SMBE Tri-National Young Investigators' Workshop 2005. Lineage-specific expansions and contractions of the bitter taste receptor gene repertoire in vertebrates. Mol. Biol. Evol. 23: 964-972.

Green MJB (1986). The distribution, status and conservation of the Himalayan musk deer Moschus chrysogaster. Biol. Conserv. 35: 347-375.

Groves CP, Wang Y and Grubb P (1994). Taxonomy of musk-deer, genus Moschus (Moschidae, Mammalia). Acta Theriol. Sin. 15: 181-197.

Guha S, Goyal SP and Kashyap VK (2007). Molecular phylogeny of musk deer: a genomic view with mitochondrial 16S rRNA and cytochrome b gene. Mol. Phylogenet. Evol. 42: 585-597.

Hilliard MA, Bergamasco C, Arbucci S, Plasterk RH, et al. (2004). Worms taste bitter: ASH neurons, QUI-1, GPA-3 and ODR-3 mediate quinine avoidance in Caenorhabditis elegans. EMBO J. 23: 1101-1111.

Hu LL and Shi P (2013). Smallest bitter taste receptor (T2Rs) gene repertoire in carnivores. Dongwuxue Yanjiu 34: E75-81.

Huang BJ, Sheng HL, XU HF, Huang B, et al. (1998). Nutrition influence on musk secretion of breeding musk deer. Zoo. Res. 19: 296-300.

Li M, Li YG and Sheng HL (1999). Taxonomic status of Anhui musk deer revisited. Chin. Sci. Bull. 44: 188-192.

Lindemann B (2001). Receptors and transduction in taste. Nature 413: 219-225.

Matsunami H, Montmayeur JP and Buck LB (2000). A family of candidate taste receptors in human and mouse. Nature 404: 601-604

Meng X, Yang O, Feng Z, Xia L, et al (2003). Timing and synchrony of parturition in Alpine musk deer (Moschus syfanicus). Folia Zoologica-praha. 52: 39-50.

Meng XX, Zhou CQ, Hu JC, Li C, et al. (2006). Musk deer farming in China. Animal Sci. 82: 1-6.

Nelson G, Chandrashekar J, Hoon MA, Feng L, et al. (2002). An amino-acid taste receptor. Nature 416: $199-202$.

Parry CM, Erkner A and le Coutre J (2004). Divergence of T2R chemosensory receptor families in humans, bonobos, and chimpanzees. Proc. Natl. Acad. Sci. U S A 101: 14830-14834.

Pietro FD, Ortenzi F, Tilio M, Concetti F, et al. (2011). Genomic DNA extraction from whole blood stored from 15 to 30 -years at $-20^{\circ} \mathrm{C}$ by rapid phenol-chloroform protocol: A useful tool for genetic epidemiology studies. Mol. Cell Probe. 25:44-48.

Sheng HL and Liu ZX (2007). The musk deer in China. The Shanghai Scientific \& Technical Publishers, Shanghai.

Shi P, Zhang J, Yang H and Zhang YP (2003). Adaptive diversification of bitter taste receptor genes in Mammalian evolution. Mol. Biol. Evol. 20: 805-814.

Shi P and Zhang J (2006). Contrasting modes of evolution between vertebrate sweet/umami receptor genes and bitter receptor genes. Mol. Biol. Evol. 23: 292-300.

Striem BJ, Pace U, Zehavi U, Naim M, et al. (1989). Sweet tastants stimulate adenylate cyclase coupled to GTP-binding protein in rat tongue membranes. Biochem. J. 260: 121-126.

Su B, Wang YX and Wang QS (2001). Mitochondrial DNA sequences imply Anhui musk deer a valid species in genus Moschus. Zoo. Res. 22: 169-173.

Tamura K, Dudley J, Nei M and Kumar S (2007). MEGA4: Molecular Evolutionary Genetics Analysis (MEGA) software version 4.0. Mol. Biol. Evol. 24: 1596-1599.

Teeter JH, Kumazawa T, Brand JG, Kalinoski DL, et al. (1992). Amino acid receptor channels in taste cells. Soc. Gen. Physiol. Ser. 47: 291-306.

Ueno A, Ueno $\mathrm{Y}$ and Tomonaga M (2004). Facial responses to four basic tastes in newborn rhesus macaques (Macaca mulatta) and chimpanzees (Pan troglodytes). Behav. Brain. Res. 154: 261-271.

Wang X, Thomas SD and Zhang J (2004). Relaxation of selective constraint and loss of function in the evolution of human bitter taste receptor genes. Hum. Mol. Genet. 13: 2671-2678.

Wu SV, Chen MC and Rozengurt E (2005). Genomic organization, expression, and function of bitter taste receptors (T2R) in mouse and rat. Physiol. Genomics 22: 139-149.

Xu Z and Xu H (2003). Population characteristics and fawn survival in musk deer (Moshus moschiferus). Acta Theriol. Sin. 23: $17-20$.

Yang Q, Meng X, Xia L and Feng Z (2003). Conservation status and causes of decline of musk deer (Moschus spp.) in China. 
Biol. Conserv. 109: 333-342.

Yarmolinsky DA, Zuker CS and Ryba NJ (2009). Common sense about taste: from mammals to insects. Cell 139: 234-244.

Yin S and W Dai (1991). A study on the secondary musk secretion of male musk deer induced physiologcally by androgen within successive two years. Acta Theriolog. Sin. 11: 9-12.

Zhang Y, Hoon MA, Chandrashekar J, Mueller KL, et al. (2003). Coding of sweet, bitter, and umami tastes: different receptor cells sharing similar signaling pathways. Cell 112: 293-301.

Zou F, Yue B, Xu L and Zhang Y (2005). Isolation and characterization of microsatellite loci from forest musk deer (Moschus berezovskii). Zool. Sci. 22: 593-598. 